\title{
Molecular Cloning and Characterization of Five Glutathione S-Transferase Genes and Promoters from Micromelalopha troglodyta (Graeser) (Lepidoptera: Notodontidae) and Their Response to Tannic Acid Stress
}

\author{
Fang Tang ${ }^{1,2, *}$, Huizhen Tu ${ }^{1,2}$, Qingli Shang ${ }^{3} \mathbb{C}$, Xiwu Gao $^{4}\left(\mathbb{D}\right.$ and Pei Liang ${ }^{4}$ \\ 1 Co-Innovation Center for Sustainable Forestry in Southern China, Nanjing Forestry University, \\ Nanjing 210037, China; tuhuizhen@live.cn \\ 2 College of Forestry, Nanjing Forestry University, Nanjing 210037, China \\ 3 School of Agricultural Science, Zhengzhou University, Zhengzhou 450001, China; shangqingli@163.com \\ 4 Department of Entomology, China Agricultural University, Beijing 100193, China; \\ gaoxiwu@263.net.cn (X.G.); liangcau@cau.edu.cn (P.L.) \\ * Correspondence: tangfang76@foxmail.com; Tel.: +86-025-8542-7794
}

Received: 16 April 2020; Accepted: 27 May 2020; Published: 1 June 2020

\begin{abstract}
Plants accumulate phenolic compounds such as tannic acid to resist insect herbivores. The survival of insects exposed to toxic secondary metabolites depends on the detoxification metabolism mediated by limited groups of glutathione S-transferases (GSTs). Micromelalopha troglodyta (Graeser) (Lepidoptera: Notodontidae) is an important foliar pest of poplar trees. GSTs play an important role in xenobiotic detoxification in M. troglodyta. Five GST genes were identified in M. troglodyta and were classified into five different cytosolic GST classes, delta, omega, sigma, theta, and zeta. Real-time fluorescent quantitative polymerase chain reaction (qPCR) was used to determine the mRNA expression of the five cloned GSTs in the midguts and fat bodies of M. troglodyta. The mRNA expression of the five GSTs was significantly induced when M. troglodyta was exposed to tannic acid. To further understand the tannic acid regulatory cascade, the $5^{\prime}$-flanking promoter sequences of the five MtGSTs were isolated by genome walking methods, and the promoters were very active and induced by tannic acid. In summary, the induction of GST mRNA expression was due to the response of five MtGST promoters to tannic acid. Therefore, MtGST promoters play an important role in the regulation of GST transcription.
\end{abstract}

Keywords: Micromelalopha troglodyta; glutathione S-transferases; tannic acid; promoter; transcriptional regulation

\section{Introduction}

The rapid growth and spread of the poplar tree account for its widespread cultivation and naturalization throughout the world. The wood is commonly used as fuel, but is also used for furniture, panelling and plywood production [1]. Poplar trees are vital to the forests of China, and Micromelalopha troglodyta (Graeser) (Lepidoptera: Notodontidae) is an important foliar pest of poplar trees. Due to the high fecundity and rapid growth of this insect, infestations usually lead to huge economic losses. Because of many kinds of plant secondary metabolites in poplars [1], the host chemical defence was also the main pressure for these pests in addition to the pesticide stress. Detoxification enzymes, including glutathione S-transferases (GSTs; EC2.5.1.18), are important parts of adaptation mechanisms. GSTs act in physiological roles by initiating the detoxication of potential alkylating agents and interacting 
with kinases, and they simultaneously bind a wide range of endogenous and exogenous ligands in non-catalytic roles [2-5]. GSTs are classified as cytosolic, mitochondrial and microsomal GSTs, and insect cytosolic GSTs are further classified into six different classes, including sigma, delta, epsilon, zeta, theta and omega [6,7]. Members of the delta and epsilon classes have been implicated in resistance to pesticides, e.g., organophosphates, organochlorines and pyrethroids [8], while the omega, theta and zeta classes appear to be involved in other cellular processes, including protection against oxidative stress [9].

The presence of plant secondary metabolites in particular plant families may be one factor mediating co-evolution between plants and their related insects [2]. The relationship between plant secondary metabolites and insect GSTs is complex. On the one hand insect GSTs may be involved in detoxifying plant secondary metabolites that are produced during feeding, but on the other, plant secondary plant compounds are also inducers of GSTs in vivo $[10,11]$. Robert et al. reported that the transcripts and accumulation of proteins of the DpGSTs were increased in Dendroctonus ponderosae fed with fresh phloem [12,13]; Dai et al. found that the transcripts of DaGSTs1 and DaGSTs2 were upregulated after feeding on host phloem in male adults of Dendroctonus armand [14]; Huang et al. showed that the expression of GST genes were induced by xenobiotic compounds and bacteria [15].

In gene expression and regulation, the promoter acts as an important cis-acting element and is also part of one of the most significant regulatory mechanisms in gene transcription, able to limit the temporal and spatial regulation of gene expression [16]. To understand molecular mechanisms, many promoters have been identified. For example, the Slgste 1 promoter was found to play an important role in regulating gene expression in response to phytochemicals and insecticides in Spodoptera litura [17]; in the promoter region of the SeGST gene from Spodoptera exigua, cis-acting elements responded to chlorpyrifos and cypermethrin [18].

Tannic acid, a plant secondary metabolite in poplars, is a plant polyphenol that is commonly distributed in many plants. As a plant allelochemical, tannic acid causes various types of effects in animals [19]. In vitro tannic acid has an inhibitory effect on GSTs. Zhang and Das reported that plant polyphenols inhibited the rat liver GST [20]; Tang et al. showed that tannic acid inhibited GST activity in two moth species [21]. However, in vivo GSTs in insects have been considered to be responsible for the detoxification of and recovery from oxidative stress agents [22]. The induction of GSTs is a mechanism by which organisms adapt to chemical stress. Chen et al. showed that GST activities were induced by tannic acid in Helicoverpa armigera (Hübner) [23]. We reported that GST activities were induced by tannic acid, and cloned the first GST gene in M. troglodyta, MtGSTd1 [19].

Little is known about GSTs in M. troglodyta, therefore, we have chosen to investigate them in our laboratory. The tissue-specific expression of the GSTs of M. troglodyta has been studied, and the midguts and fat bodies have higher GST activities than the head and integument [24]. In vivo GST activities were induced by tannic acid in M. troglodyta [25]. Furthermore, MtGSTd1, the first GST gene identified in M. troglodyta, was cloned and characterized in our laboratory [19]. However, there have been no previous reports on the regulatory mechanism of GST expression in M. troglodyta. To understand the regulatory mechanism controlling GST expression in M. troglodyta, we cloned 5 GST genes and $5 \mathrm{MtGST}$ promoters. Then, we assessed the response of mRNA expression of 5 GST genes to tannic acid and measured the response of $5 \mathrm{MtGST}$ promoters to tannic acid. We address the following questions in this study: (1) How does tannic acid affect the expression of MtGST mRNA and (2) how do the $5 \mathrm{MtGST}$ promoters respond to tannic acid? These studies are very important in understanding the regulatory mechanism of MtGST mRNA expression in M. troglodyta. These data are important for understanding the tannic acid regulatory cascade and for the development of an effective integrated pest management program for M. troglodyta. 


\section{Materials and Methods}

\subsection{Insect and Cell Cultures}

The larvae used in these assays were from a colony initially established by collecting M. troglodyta from poplar trees in Nanjing ( $\left.31^{\circ} 56^{\prime} 17.00^{\prime \prime} \mathrm{N}, 118^{\circ} 22^{\prime} 35.98^{\prime \prime} \mathrm{E}\right)$, Jiangsu Province, China. Larvae were supplied fresh poplar leaves in a room maintained at $26 \pm 1{ }^{\circ} \mathrm{C}$ and $70 \%-80 \%$ relative humidity with a photoperiod of 16:8 (light: dark). The induction of GST mRNA expression by tannic acid was studied using larvae feeding on poplar leaves that had been immersed in tannic acid solutions. Tannic acid (Sigma Chemical, St. Louis, MO, USA) was dissolved in a small aliquot of ethanol and then serially diluted in distilled water to test concentrations of $0.001,0.01,0.1,1$, and $10 \mathrm{mg} / \mathrm{mL}$. Freshly collected poplar leaves were immersed in the various solutions for $10 \mathrm{~s}$ respectively and then allowed to air-dry. Once dried, two treated leaves were placed into triangular bottles with 5 newly molted third instars, and 10 triangular bottles were set up per concentration, then placed on a shelf in a rearing room. Controls consisted of larvae feeding on leaves immersed in distilled water. Larvae were allowed to feed on treated leaves for $96 \mathrm{~h}$, and then they were collected for dissection. The larvae of $M$. troglodyta were dissected, and the fat bodies and midguts were separated on ice. Firstly, the fat bodies were collected. Secondly, after the peritrophic membrane containing midgut contents were removed, midguts were washed in $1.15 \%$ ice-cold $\mathrm{KCl}$ and collected. All experiments were independently conducted three times.

Sf9 cells were routinely cultured in SF-900 II serum-free medium (Invitrogen, Carlsbad, CA, USA) supplemented with 10\% heat-inactivated fetal bovine serum (HyClone-QB Perbio, Logan, UT, USA), $50 \mathrm{U} / \mathrm{mL}$ penicillin, $50 \mu \mathrm{g} / \mathrm{mL}$ streptomycin and $12 \mu \mathrm{g} / \mathrm{mL}$ gentamycin (Invitrogen) at $28{ }^{\circ} \mathrm{C}$.

\subsection{Cloning the cDNA Sequences of the Glutathione S-Transferases (GSTs)}

Total RNA was extracted from one 5th instar larvae using TRIzol reagent (Invitrogen, Thermo Fisher Scientific, Inc., Waltham, MA, USA) according to the manufacturer's instructions. Total RNA was then treated with DNaseI (TaKaRa, China), and cDNA was synthesized using the PrimeScript 1st Strand cDNA Synthesis Kit (TaKaRa, China). Primers (F and R) were designed and used in polymerase chain reactions (PCR) to amplify the full-length open reading frame (ORF) of GSTs (Table 1). PCR was performed as follows: $94{ }^{\circ} \mathrm{C}$ for $5 \mathrm{~min}$; 30 cycles of $94{ }^{\circ} \mathrm{C}$ for $30 \mathrm{~s}, 53{ }^{\circ} \mathrm{C}$ for $30 \mathrm{~s}$, and $72{ }^{\circ} \mathrm{C}$ for $1 \mathrm{~min}$; and $72{ }^{\circ} \mathrm{C}$ for $10 \mathrm{~min}$. The PCR products were purified using the Generay DNA Recovery kit (TaKaRa). The purified cDNA fragments were ligated to pMD19-T vectors (TaKaRa) and cloned into DH5 $\alpha$ competent cells. At least three clones were sequenced by the GenScript (Nanjing, China) Biological Technology (Co., Ltd.). For each plasmid insert, both strands of DNA were sequenced at least twice.

\subsection{Sequence Identity and Phylogenetic Analysis}

The amino acid sequences of the GSTs were deduced from their cDNAs and aligned using ClustalX2. Alignments were converted to meg files using MEGA software. A phylogenetic tree was constructed by the neighbour-joining method using MEGA 6.0 [26]. 
Table 1. Primers used in experiments.

\begin{tabular}{|c|c|c|c|c|c|c|}
\hline GenBank & Gene & Primer & Sequence $\left(5^{\prime}-3^{\prime}\right)$ & $\begin{array}{c}\text { Annealing } \\
\text { Temperatures }\left({ }^{\circ} \mathrm{C}\right)\end{array}$ & $\begin{array}{l}\text { Amplicon } \\
\text { Size (bp) }\end{array}$ & Application \\
\hline \multirow{10}{*}{ KU 963403} & \multirow{10}{*}{ MtGSTd2 } & $\mathrm{F}$ & TCAGCGTTTGAAGATGTCG & \multirow{2}{*}{53} & \multirow{2}{*}{678} & ORF \\
\hline & & $\mathrm{R}$ & GCTTCTTACAGCTCGGTTTTAG & & & ORF \\
\hline & & Q-F & AAAGCCGATGAAGCCAAGTT & \multirow[b]{2}{*}{60} & \multirow[b]{2}{*}{105} & gPCR \\
\hline & & $\mathrm{Q}-\mathrm{R}$ & TGCCAGGGTCAGTTTATCTCC & & & qPCR \\
\hline & & Common 1 & GTAATACGACTCACTATAGGGC & \multirow{2}{*}{67} & \multirow{2}{*}{-} & Genome Walker \\
\hline & & 1 & ATCGTCTACTATCGTGGGAATGGTGTG & & & Genome Walker \\
\hline & & Common 2 & ACTATAGGGCACGCGTGGT & \multirow[b]{2}{*}{67} & \multirow[b]{2}{*}{975} & Genome Walker \\
\hline & & 2 & GACGAGCTTCAGGTTGAGTTGGATGT & & & Genome Walker \\
\hline & & F-1 & AACTCGAGAGATTACTATAGGGCACG & \multirow{2}{*}{62} & \multirow{2}{*}{920} & Constructs \\
\hline & & R-1 & AA $\underline{G C T A G C A G G T A G T A C A G G T C G A T C ~}$ & & & Constructs \\
\hline \multirow{10}{*}{ KU 963404} & \multirow{10}{*}{ MtGSTo1 } & $\mathrm{F}$ & TCGCTGCCATCATGTCTG & \multirow{2}{*}{53} & \multirow{2}{*}{778} & ORF \\
\hline & & $\mathrm{R}$ & GTTTATTCCTTCTTCTTCCTGG & & & ORF \\
\hline & & Q-F & ACTATACAGCTGCCTTCAACGC & \multirow[b]{2}{*}{60} & \multirow[b]{2}{*}{153} & qPCR \\
\hline & & Q-R & GCCACAATGTGAAGTCAACGAG & & & qPCR \\
\hline & & Common 1 & GTAATACGACTCACTATAGGGC & \multirow[b]{2}{*}{67} & \multirow{2}{*}{-} & Genome Walker \\
\hline & & 1 & ACTAAAACTGTTCTCTCGGCGTATGGG & & & Genome Walker \\
\hline & & Common 2 & ACTATAGGGCACGCGTGGT & \multirow{2}{*}{67} & \multirow{2}{*}{977} & Genome Walker \\
\hline & & 2 & GGGCAGAATCTCATAGCGAATACACG & & & Genome Walker \\
\hline & & F-1 & AACTCGAGATTACTATAGGGCACGC & \multirow{2}{*}{62} & \multirow[b]{2}{*}{847} & Constructs \\
\hline & & $\mathrm{R}-1$ & AAGCTAGCGGTTTGTAAATGTTTTTC & & & Constructs \\
\hline \multirow{10}{*}{ KU 963405} & \multirow{10}{*}{ MtGSTs1 } & F & GAGTCCTTGACAATGGCTA & \multirow{2}{*}{53} & \multirow{2}{*}{660} & ORF \\
\hline & & $\mathrm{R}$ & GCTCGCTATTGCACAACC & & & ORF \\
\hline & & Q-F & GACTTTTGGGCCAACATCAG & \multirow{2}{*}{60} & \multirow{2}{*}{112} & qPCR \\
\hline & & $\mathrm{Q}-\mathrm{R}$ & CAAATCTGGGCAAGAAGAACAC & & & qPCR \\
\hline & & Common 1 & GTAATACGACTCACTATAGGGC & & & Genome Walker \\
\hline & & 1 & AACCTTACATCCTCAAATTCCTGTTTAGTG & 67 & - & Genome Walker \\
\hline & & Common 2 & ACTATAGGGCACGCGTGGT & & & Genome Walker \\
\hline & & 2 & GCTAAGGCAGGTGCTTCAAAATAATACAG & 67 & 1012 & Genome Walker \\
\hline & & F-1 & AACTCGAGCGACGAAGGCTT & & & Constructs \\
\hline & & $\mathrm{R}-1$ & AAGCTAGCATTTGCTGCACTATCA & 62 & 926 & Constructs \\
\hline
\end{tabular}


Table 1. Cont.

\begin{tabular}{|c|c|c|c|c|c|c|}
\hline GenBank & Gene & Primer & Sequence $\left(5^{\prime}-3^{\prime}\right)$ & $\begin{array}{c}\text { Annealing } \\
\text { Temperatures }\left({ }^{\circ} \mathrm{C}\right)\end{array}$ & $\begin{array}{l}\text { Amplicon } \\
\text { Size (bp) }\end{array}$ & Application \\
\hline \multirow{10}{*}{ KU 963408} & \multirow{10}{*}{ MtGSTt1 } & $\mathrm{F}$ & GTTCAATACCTTCAAGTTTTTC & \multirow[b]{2}{*}{53} & \multirow{2}{*}{722} & ORF \\
\hline & & $\mathrm{R}$ & ACACTTTAGACTTAACTTTAGACTGC & & & ORF \\
\hline & & Q-F & CCACTGTCGCTGATCTGCTG & \multirow{2}{*}{60} & \multirow{2}{*}{126} & qPCR \\
\hline & & $\hat{\mathrm{Q}}-\mathrm{R}$ & AGGGGCTGAAATGTCGTTG & & & qPCR \\
\hline & & Common 1 & GTAATACGACTCACTATAGGGC & \multirow{2}{*}{67} & \multirow{2}{*}{-} & Genome Walker \\
\hline & & 1 & CAAAGTACAAGAACATAGCCGAGTGGTG & & & Genome Walker \\
\hline & & Common 2 & ACTATAGGGCACGCGTGGT & \multirow[b]{2}{*}{67} & \multirow[b]{2}{*}{1894} & Genome Walker \\
\hline & & 2 & TCTTCACAGAGTGGTCGTTATTTCAGTTC & & & Genome Walker \\
\hline & & F-1 & AACTCGAGTGCCTGCAGGTC & \multirow{2}{*}{62} & \multirow[b]{2}{*}{1853} & Constructs \\
\hline & & $\mathrm{R}-1$ & AAGCTAGCGCTTTGATTTGGTC & & & Constructs \\
\hline \multirow{10}{*}{ KU 963410} & \multirow{10}{*}{$M t G S T z 1$} & $\mathrm{~F}$ & CTCAAAATACAACGGGAACC & \multirow{2}{*}{53} & \multirow[b]{2}{*}{707} & ORF \\
\hline & & $\mathrm{R}$ & CGACCGTGACAAGAGGC & & & ORF \\
\hline & & Q-F & AGTCAATCCGATGGAGCAGG & \multirow[b]{2}{*}{60} & \multirow{2}{*}{180} & qPCR \\
\hline & & $\mathrm{Q}-\mathrm{R}$ & GGTTGGATGCCTGATGCTATT & & & gPCR \\
\hline & & Common 1 & GTAATACGACTCACTATAGGGC & \multirow{2}{*}{67} & \multirow{2}{*}{-} & Genome Walker \\
\hline & & 1 & CTTGTCTCTTCCAGGTAGTGCATTATGTTC & & & Genome Walker \\
\hline & & Common 2 & ACTATAGGGCACGCGTGGT & \multirow{2}{*}{67} & \multirow{2}{*}{1590} & Genome Walker \\
\hline & & 2 & ATGGGATCTCCTTCAAGTTGAGTGCG & & & Genome Walker \\
\hline & & F-1 & AACTCGAGGGCACGCGTG & \multirow{2}{*}{62} & \multirow[b]{2}{*}{1177} & Constructs \\
\hline & & $\mathrm{R}-1$ & AAGCTAGCTTGTAAGTCGGTATGTATGTAA & & & Constructs \\
\hline \multirow{2}{*}{ GU 262991} & \multirow{2}{*}{ Actin } & Q-F & CTCTGGTCGACTTGAGGCTGGAC & \multirow{2}{*}{60} & \multirow{2}{*}{241} & qPCR \\
\hline & & $\hat{Q}-\mathrm{R}$ & CTCTGGTCGACTTGAGGCTGGAC & & & qPCR \\
\hline
\end{tabular}

Primers were designed using Premier 5.0 software and synthesized by Shanghai Generay Biotechnology Co., Ltd. F, forward; R, reverse. Enzyme cutting sites are underlined. 


\subsection{Induction of GST Transcription in Micromelalopha troglodyta by Tannic Acid}

Quantitative real-time PCR (qPCR) was performed on a 7500 Real-Time PCR system (Applied Biosystems, Foster, CA) to compare the expression of MtGST mRNA in the midguts and fat bodies of M. troglodyta larvae exposed to tannic acid and the control. Total RNA for expression analysis was extracted from $50 \mathrm{mg}$ midguts or fat bodies. The integrity and quality of total RNA were tested by running 1\% agarose gel electrophoresis and measuring absorbance at 260 and $280 \mathrm{~nm}$ using a Thermo Scientific NanoDrop2000. According to the instructions of PrimeScript RT reagent Kit with gDNA Eraser, $5 \mu \mathrm{g}$ total RNA was used to reverse-transcribe into cDNA and then stored at $-80{ }^{\circ} \mathrm{C}$ or used for the determination of GST mRNA expression. MtGST mRNA expression was studied using the SYBR $^{\circledR}$ Premix Ex Taq ${ }^{\mathrm{TM}}$ II (Tli RNaseH Plus) kit (TaKaRa Biotechnology (Dalian) Co., Ltd.). Primers (Q-F and Q-R) were designed based on the GST sequences (Table 1). The specificity and sensitivity of these primers were evaluated through melting curve analysis, and the amplification efficiencies were calculated from the standard curves. The amplification efficiency of each pair of primer used in qPCR was within the range of $90 \%-105 \%$. The amplification of cDNA by qPCR was performed in a $20 \mu \mathrm{L}$ mixture that contained approximately $1 \mu \mathrm{L}$ of cDNA, $10 \mu \mathrm{L}$ of SYBR Premix Ex Taq, $0.4 \mu \mathrm{L}$ of Rox reference dye (503), $0.4 \mu \mathrm{L}$ of both sense primer $(10 \mu \mathrm{M})$ and antisense primer $(10 \mu \mathrm{M})$ of GST, and $7.8 \mu \mathrm{L}$ of double-distilled water. Actin was used as an internal standard $(0.4 \mu \mathrm{L}$ for each). The qPCR conditions were as follows: $95^{\circ} \mathrm{C}$ for $30 \mathrm{~s}$ followed by 40 cycles of $95^{\circ} \mathrm{C}$ for $5 \mathrm{~s}$ and $60^{\circ} \mathrm{C}$ for $34 \mathrm{~s}$. To confirm the amplification of specific products, melting-curve cycles were performed with the following parameters: $95^{\circ} \mathrm{C}$ for $15 \mathrm{~s}, 60^{\circ} \mathrm{C}$ for $1 \mathrm{~min}$, and $95^{\circ} \mathrm{C}$ for $15 \mathrm{~s}$. All experiments were independently conducted four times. The transcript levels of the target genes were expressed as normalized transcript abundances using actin as the internal reference gene. Relative gene expression was calculated using the $2^{-\Delta \Delta C t}$ method [27].

\subsection{Cloning the Sequences of the Five GST Promoter Genes}

Genomic DNA was extracted from individual larvae using DNA isolation reagent (TaKaRa, China). The promoters were cloned using the Genome Walker ${ }^{\mathrm{TM}}$ Universal kit (Clontech, Mountain View, CA, USA). Primers (1 and 2) were designed according to the manufacturer's instructions (Table 1). A two-step PCR was performed as follows: $94{ }^{\circ} \mathrm{C}$ for $5 \mathrm{~min} ; 7$ cycles of $94{ }^{\circ} \mathrm{C}$ for $25 \mathrm{~s}$ and $72{ }^{\circ} \mathrm{C}$ for $3 \mathrm{~min} ; 32$ cycles of $94^{\circ} \mathrm{C}$ for $25 \mathrm{~s}$ and $67^{\circ} \mathrm{C}$ for $3 \mathrm{~min} ; 67^{\circ} \mathrm{C}$ for $7 \mathrm{~min} ; 94^{\circ} \mathrm{C}$ for $5 \mathrm{~min} ; 5$ cycles of $94^{\circ} \mathrm{C}$ for $25 \mathrm{~s}$ and $72{ }^{\circ} \mathrm{C}$ for $3 \mathrm{~min} ; 30$ cycles of $94^{\circ} \mathrm{C}$ for $25 \mathrm{~s}$ and $67^{\circ} \mathrm{C}$ for $3 \mathrm{~min}$; followed by $67^{\circ} \mathrm{C}$ for $7 \mathrm{~min}$.

The PCR products were cloned into the pMD-19T vector (TaKaRa, China), transformed into DH5a competent cells and sequenced by the Nanjing GenScript Biotechnology Company. The searches for homologous sequences were performed using BLASTN against the National Center for Biotechnology Information database (http://www.ncbi.nlm.nih.gov/).

\subsection{Analysing the Sequences of the Five GST Promoter Genes}

The promoter predictions for the sequences with a score cut-off of 0.80 were conducted with the Berkeley Drosophila Genome Project (BDGP) database (http://www.fruitfly.org/seq_tools/ promoter.html). The transcription factor binding sites were predicted by constructing matrices on the fly from TRANSFAC 4.0 sites using TRANSFAC 4.0 software on the AliBaba 2.1 database (http:// www.gene-regulation.com/pub/programs/alibaba2/index. html) with specific parameters (Pairsim to know sites value is 64 , match width in bp value is 10 , minimum number of sites is 5 , minimum match conservation value is $75 \%$, similarity of sequence to match value is $100 \%$, and factor class level is 4 ).

\subsection{Construction of the MtGSTd2 Promoter-PGL 4.10, MtGST01 Promoter-PGL 4.10, MtGSTs1} Promoter-PGL 4.10, MtGSTt1 Promoter-PGL 4.10 and MtGSTz1 Promoter-PGL 4.10 Constructs

To generate a construct fusing the MtGST promoters with the luciferase reporter gene $l u c 2$ (Photinus pyralis), primers (F-1 and R-1) with Xho I and Nhe I sites were designed based on the promoter 
sequence (Table 1). The PCR products and reporter genes were digested by Xho I and Nhe I digestion. Thus, five recombinant plasmids, MtGSTd2 promoter-PGL 4.10, MtGSTo1 promoter-PGL 4.10, MtGSTs1 promoter-PGL 4.10, MtGSTt1 promoter-PGL 4.10 and MtGSTz1 promoter-PGL 4.10, were obtained.

\subsection{Transient Transfection and Dual Luciferase Assay}

Sf9 cells were seeded onto a 96-well plate $\left(9 \times 10^{4}\right.$ cells/well) and transiently cotransfected with MtGST promoter-PGL 4.10 luciferase reporter constructs (200 ng/well) and the internal renilla luciferase reporter plasmid phRL-TK (Promega; 20 ng/well) using Cellfectin II reagent (Invitrogen; $1 \mu \mathrm{L} /$ well). The transfection efficiency and luciferase activity were determined as described by Peng et al. [28]. After $48 \mathrm{~h}$, the cells were harvested, and the resulting lysates were used to measure the renilla and firefly luciferase activities with a Dual-Glo ${ }^{\circledR}$ Luciferase Assay System (Promega) on an FLx800 ${ }^{\mathrm{TM}}$ fluorescence microplate reader (Biotek, Winooski, VT, USA). The relative firefly luciferase activity was normalized against the renilla luciferase activity.

The tannic acid was dissolved in a small aliquot of acetone and then serially diluted in acetone to test concentrations of $0.01,0.1$ and $1 \mathrm{mg} / \mathrm{mL}$. Sf 9 cells were seeded onto a 96 -well plate $\left(9 \times 10^{4}\right.$ cells/well $)$ and transiently cotransfected with MtGST promoter-PGL 4.10 luciferase reporter constructs (200 ng/well) and the internal renilla luciferase reporter plasmid phRL-TK (Promega; $20 \mathrm{ng} /$ well) using Cellfectin II reagent (Invitrogen; $1 \mu \mathrm{L} /$ well). Five hours post-transfection, tannic acid or equal volumes of acetone were added to the wells. After $48 \mathrm{~h}$, the cells were harvested, and the resulting lysates were used to measure the renilla and firefly luciferase activities with a Dual-Glo ${ }^{\circledR}$ Luciferase Assay System (Promega) on an FLx800 ${ }^{\mathrm{TM}}$ fluorescence microplate reader (Biotek, Winooski, VT, USA). The relative firefly luciferase activity was normalized against the renilla luciferase activity reported for each construct. The induction folds reported are expressed as a ratio of the normalized tannic acid-induced firefly luciferase activity to the normalized basal firefly luciferase activity (acetone control).

\subsection{Data Analysis}

Data collected from these assays were subjected to analysis of variance using InStat software (GraphPad, San Diego, CA, USA). A Student's $t$-test followed by a two-tailed unpaired $t$-test was used to compare the significant differences of all two-sample. The statistical significance of multiple sample comparisons was calculated using a one-way analysis of variance followed by Tukey's multiple comparisons. A value of $P<0.05$ was considered statistically significant.

\section{Results}

\subsection{Cloning and Identity of the GST cDNA}

Five different GST genes were identified in M. troglodyta. These GST cDNA sequences and their deduced amino acid sequences were deposited in GenBank with the following accession numbers: KU 963403, KU 963404, KU 963405, KU 963408 and KU 963410. The identities of these GST genes were revealed by a BLASTX search against the NCBI non-redundant database, and the optimal alignment of cloned sequences for Blastp is summarized in Supplementary Table S1. A phylogenetic analysis of the five GSTs deduced from their cDNA revealed that the GSTs belonged to five different cytosolic classes, delta (MtGSTd2), omega (MtGSTo1), sigma (MtGSTs1), theta (MtGSTt1), and zeta (MtGSTz1). The assignment of the five GSTs to the five classes was clearly supported by sequence similarity analysis. Fifty-five GST genes from 23 species, such as Spodoptera litura, Papilio xuthus, Bombyx mori and Cnaphalocrocis medinalis, showed different degrees of genetic relationships with the five GST genes. The percentages of identity of the deduced amino acids for the five GSTs were $43 \%-95 \%$ relative to other 55 GST genes established in the phylogenetic tree, and MtGSTd2 showed the strongest genetic relationship with GSTd of Bombyx mori and Spodoptera litura. MtGSTo1 was closely related to CsGSTo1, MtGSTs1 shared the highest identity with CfGST, and MtGSTt1 and MtGSTz1 shared the highest identity with SIGSTt1 and SIGSTz1, respectively (Figure 1). 


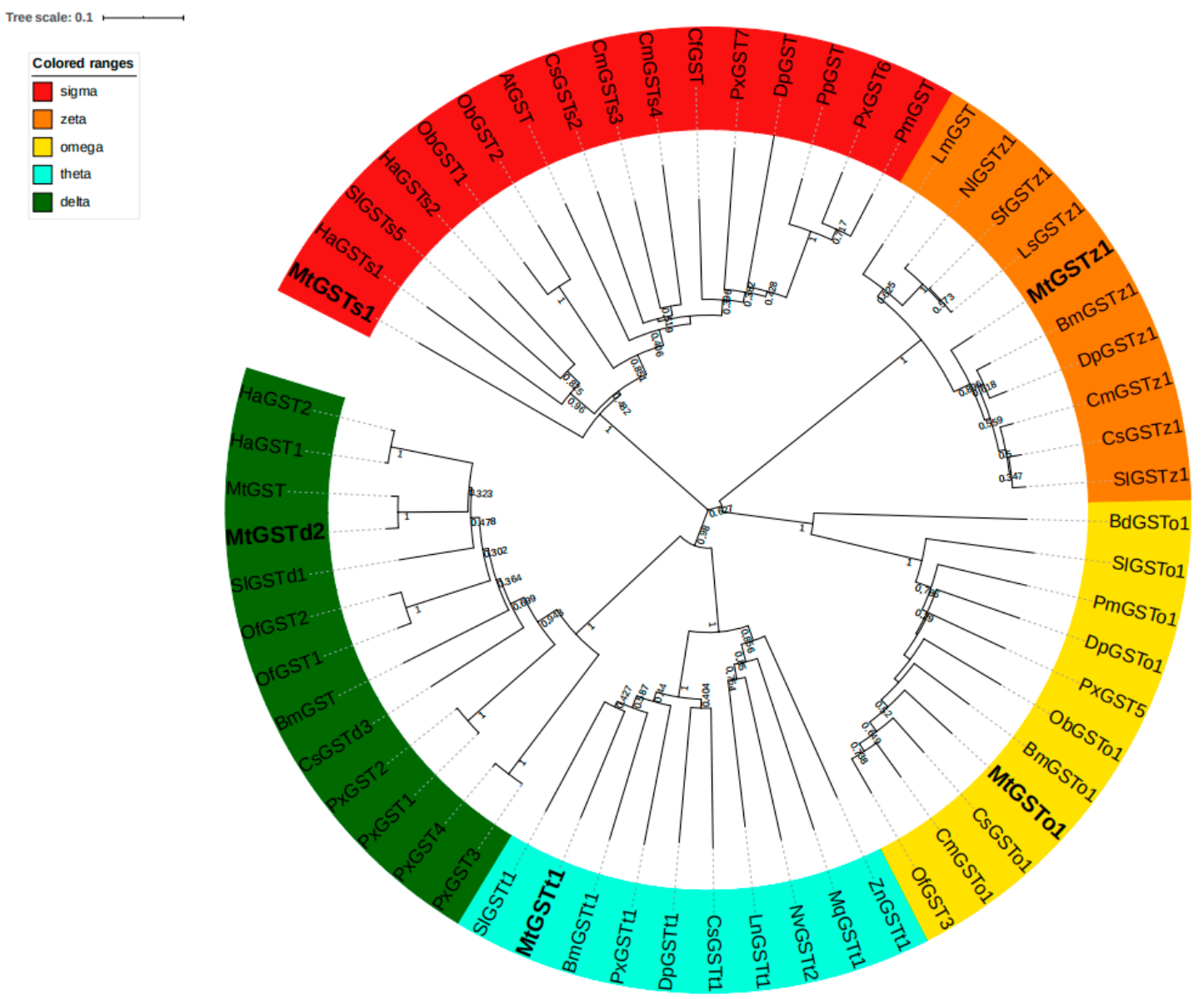

Figure 1. Phylogenetic relationships of 5 glutathione S-transferase (GST) genes of M. troglodyta with 55 GST proteins from 23 species.

The phylogenetic tree was constructed using the neighbor joining method (NJ), and the P-distances modeling and a pairwise deletion of gaps were performed by the MEGA 6.0 software package. The reliability of the tree structure and node support was evaluated by bootstrap analysis with 1000 replicates. The GenBank accession numbers of the sequences used in this tree are listed in Table S2.

At: Amyelois transitella; Bd: Bactrocera dorsalis; Bm: Bombyx mori; Cf: Choristoneura fumiferana; $\mathrm{Cm}$ : Cnaphalocrocis medinalis; Cs: Chilo suppressalis; Dp: Danaus plexippus; Ha: Helicoverpa armigera; Lm: Locusta migratoria; Ln: Lasius niger; Ls: Laodelphax striatella; Mq: Melipona quadrifasciata; Mt: Micromelalopha troglodyta; Nl: Nilaparvata lugens; Nv: Nasonia vitripennis; Ob: Operophtera brumata; Of: Ostrinia furnacalis; Pm: Papilio machaon; Pp: Papilio polytes; Px: Papilio xuthus; Sf: Sogatella furcifera; Sl: Spodoptera litura; Zn: Zootermopsis nevadensis.

\subsection{Induction of GST Gene Expression in M. troglodyta by Tannic Acid}

The expression of the five GST mRNAs in the midguts and fat bodies of M. troglodyta larvae was clearly induced by exposure to five different concentrations of tannic acid, $0.001,0.01,0.1,1$, and $10 \mathrm{mg} / \mathrm{mL}$, for $96 \mathrm{~h}$ (Figure 2). The effects of tannic acid on the mRNA expression of GST genes at $96 \mathrm{~h}$ were compared with those of the double-distilled water treatment (control) and the treatments with different concentrations of tannic acid at $96 \mathrm{~h}$ post-treatment (Figure 2). A statistical analysis of the expression results showed that some differences were significant. For $M t G S T d 2,0.01 \mathrm{mg} / \mathrm{mL}$ tannic acid increased while other treatments reduced the expression in midguts $(\mathrm{F}=164.30 ; \mathrm{df}=5,18 ; P<0.0001)$, and 1 and $10 \mathrm{mg} / \mathrm{mL}$ tannic acid increased while other treatments reduced the expression in fat bodies $(\mathrm{F}=81.07 ; \mathrm{df}=5,18 ; P<0.0001)$. The mRNA expression of $M t G S T o 1$ was significantly upregulated at a concentration of $0.01-1 \mathrm{mg} / \mathrm{mL}$, whereas other concentrations of tannic acid caused significant 
downregulation in midguts $(\mathrm{F}=448.22 ; \mathrm{df}=5,18 ; P<0.0001)$; the mRNA expression of $M t G S T o 1$ was significantly upregulated at a concentration of $10 \mathrm{mg} / \mathrm{mL}$, whereas $0.01-1 \mathrm{mg} / \mathrm{mL}$ tannic acid caused significant downregulation in fat bodies $(\mathrm{F}=10192 ; \mathrm{df}=5,18 ; P<0.0001)$. All five concentrations of tannic acid significantly induced the expression of MtGSTs1 in midguts $(\mathrm{F}=745.80 ; \mathrm{df}=5,18$; $P<0.0001$ ), and 1 and $10 \mathrm{mg} / \mathrm{mL}$ tannic acid induced the expression of MtGSTs1 mRNA in the fat bodies $(\mathrm{F}=19.153 ; \mathrm{df}=5,18 ; P<0.0001)$. Tannic acid caused significant downregulation of $M t G S T t 1$ mRNA expression at concentrations of $0.001-0.1 \mathrm{mg} / \mathrm{mL}(\mathrm{F}=47.67 ; \mathrm{df}=5,18 ; P<0.0001)$, and all five concentrations of tannic acid decreased MtGSTt1 mRNA expression in fat bodies $(\mathrm{F}=8350.8 ; \mathrm{df}=5,18$; $P<0.0001)$. The expression of MtGSTz1 mRNA was significant downregulated by $0.001,0.01$ and $10 \mathrm{mg} / \mathrm{mL}$ tannic acid in midguts $(\mathrm{F}=41.30 ; \mathrm{df}=5,18 ; P<0.0001)$; the expression of MtGSTz1 mRNA was significantly upregulated by $0.1-10 \mathrm{mg} / \mathrm{mL}$ tannic acid $(\mathrm{F}=48.32 ; \mathrm{df}=5,18 ; P<0.0001)$ (Figure 2).

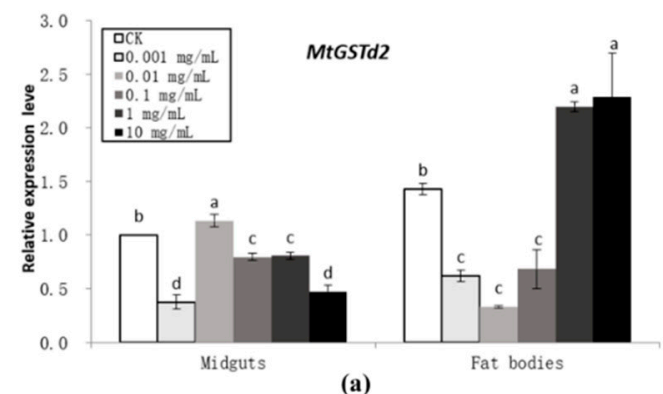

(a)

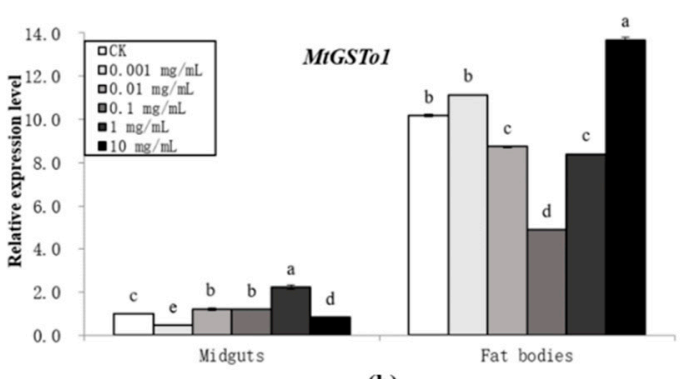

(b)

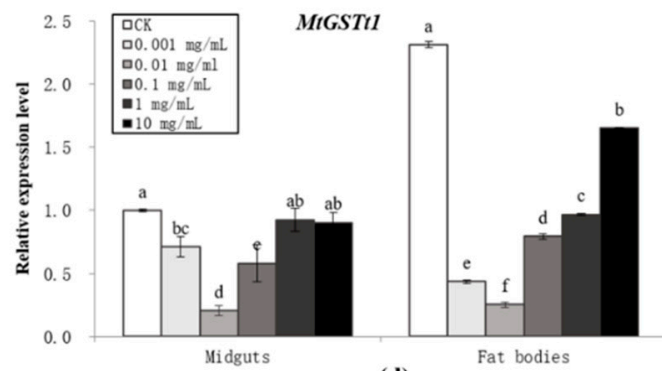

(d)

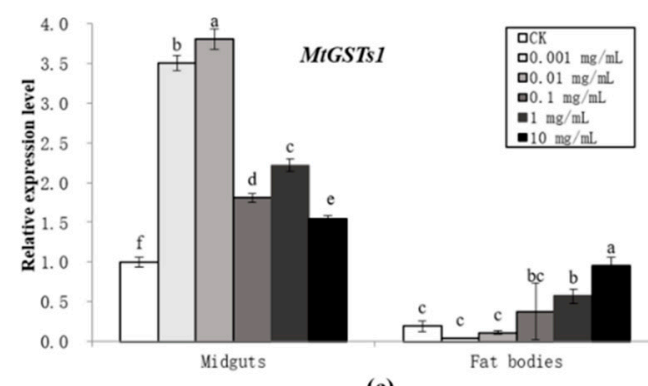

(c)

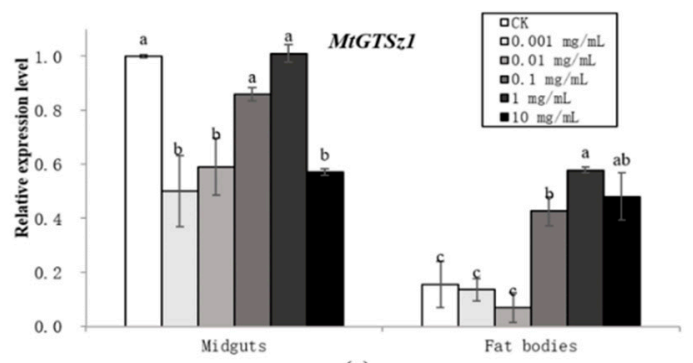

(e)

Figure 2. Quantification of relative expression of the five GST genes in M. troglodyta exposed to double-distilled water as a control (0) and to tannic acid at five different concentrations $(0.001,0.01$, $0.1,1$, and $10 \mathrm{mg} / \mathrm{mL}$ ) at $96 \mathrm{~h}$ posttreatment (a) MtGSTd2; (b) MtGSTo1; (c) MtGSTs1; (d) MtGSTt1; (e) MtGSTz1.

The mRNA levels in the control and each treatment were normalized using to the mRNA level of actin, the reference gene. The mean expression in each treatment was shown as the fold change from the mean expression in the control. The vertical bars indicate standard deviations of the mean $(n=4)$. The statistical significance of the gene expressions was calculated using a one-way analysis of variance followed by Tukey's multiple comparisons; a Student's $t$-test followed by a two-tailed unpaired $t$-test 
was used to compare the significant differences between midguts and fat bodies. A value of $P<0.05$ was considered statistically significant.

In this study, tissue-specific expression patterns of the genes were analysed. All five GST genes had different expression levels between midguts and fat bodies. MtGSTd2 $(\mathrm{t}=15.93 ; \mathrm{df}=6 ; P<0.0001)$, MtGSTo1 $(\mathrm{t}=298.27 ; \mathrm{df}=6 ; P<0.0001)$ and MtGSTt1 $(\mathrm{t}=97.30 ; \mathrm{df}=6 ; P<0.0001)$ showed higher expression levels in fat bodies; however, MtGSTs1 $(\mathrm{t}=17.63 ; \mathrm{df}=6 ; P<0.0001)$ and $M t G S T z 1(\mathrm{t}=19.63$; $\mathrm{df}=6 ; P<0.0001)$ had higher expression in midguts. For instance, the expression of MtGSTo1 in fat bodies was 10 times higher than that in midguts, and MtGSTz1 expression was more than 6 times higher in midguts than in fat bodies (Figure 2).

\subsection{Characterization of the 5'-Flanking Promoter Sequences of the Five MtGST Genes}

Sequences of the upstream portion of the 5 MtGSTs were obtained by genome walking methods (Figures S1-S5). Sequence analysis showed that the terminal sequences of these clones were identical to the MtGST cDNA sequences, which confirmed that the correct fragments of the upstream sequences of the five MtGSTs had been amplified. Software was used to analyse the upstream sequences and predict the locations of the transcription start sites. The nucleotides were numbered relative to the transcription start sites (TSSs) indicated by +1 , with upstream sequences preceded by a ' - ' and downstream sequences preceded by a ' + '. Based on sequence analysis, the typical characteristics were predicted, such as the recognition site CCAAT/enhancer-binding protein alpha (C/EBPalp).

\subsection{Functional Analysis of MtGST Promoters}

The restructured plasmids were transferred into Sf9 cells by lipofectin-mediated transfection and luciferase assays were performed. At $48 \mathrm{~h}$ after transfection, MtGSTd2 showed the highest promoter strength, which was more than 15 times that of the control. MtGSTo1 and MtGSTs1 exhibited promoter activity, albeit at weaker levels than MtGSTd2, but still showed strong promoter activity in Sf9 cells. There was no significant difference between MtGSTt1, MtGSTz1 and the control promoter strengths (Figure 3).

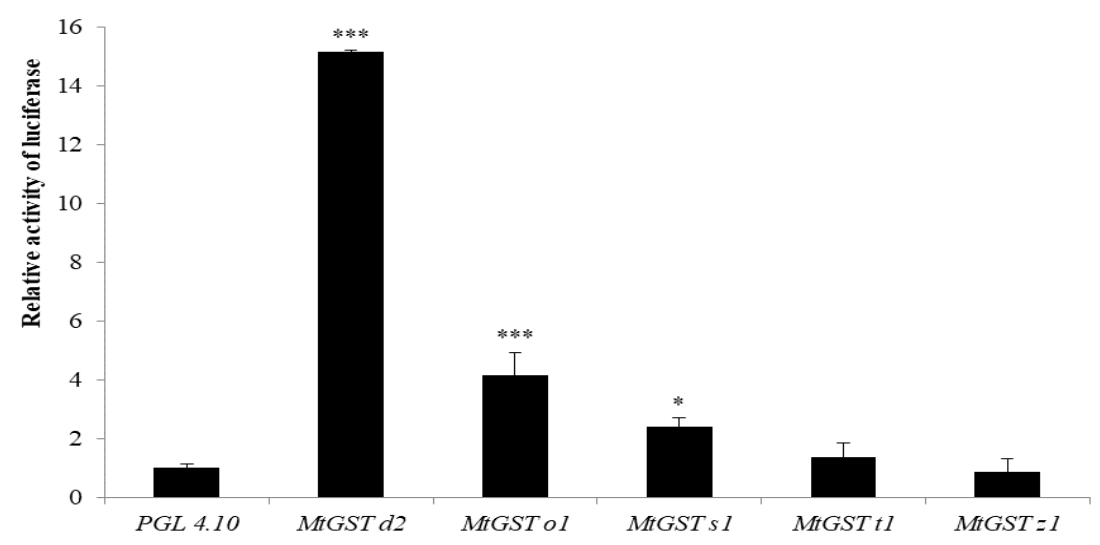

Figure 3. The promoter activity was measured as luciferase activity in Sf9 cells. ${ }^{*}$ on the bars indicate that the means are significantly different between the control and MtGST promoters ( ${ }^{* * *}$ means $P<0.001$, * means $P<0.05)$.

The relative luciferase activity of PGL 4.10 was defined as 1, and the PGL 4.10 promoter was used as a positive control. The vertical bars indicate standard deviations of the mean $(n=4)$.

The restructured plasmids were transferred into Sf9 cells by lipofectin-mediated transfection, and luciferase assays were performed in the presence of tannic acid. At $48 \mathrm{~h}$ after transfection, the MtGSTd2 ( $\mathrm{F}=14.49 ; \mathrm{df}=3,12 ; P=0.0003), M t G S T 01(\mathrm{~F}=10.09 ; \mathrm{df}=3,12 ; P=0.0013)$ and MtGSTt1 $(\mathrm{F}=33.48 ; \mathrm{df}=3,12 ; P<0.0001)$ promoter activities were induced by 0.01 and $0.1 \mathrm{mg} / \mathrm{mL}$ tannic acid, 
and the MtGSTs1 ( $\mathrm{F}=8.66 ; \mathrm{df}=3,12 ; P=0.0025)$ and MtGSTz1 $(\mathrm{F}=5.56 ; \mathrm{df}=3,12 ; P=0.0126)$ promoter activities were increased by 0.01 or $0.1 \mathrm{mg} / \mathrm{mL}$ tannic acid, respectively (Figure 4 ).

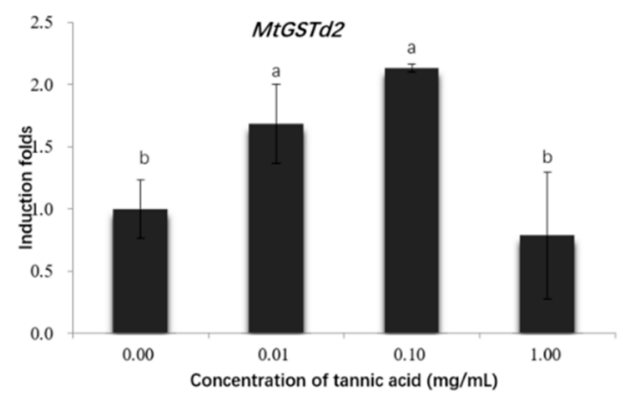

(a)

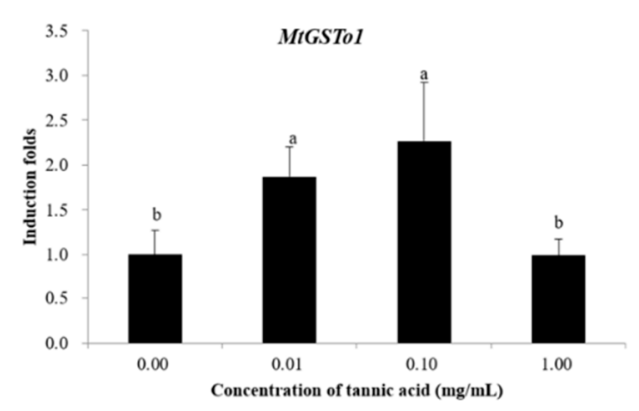

(b)

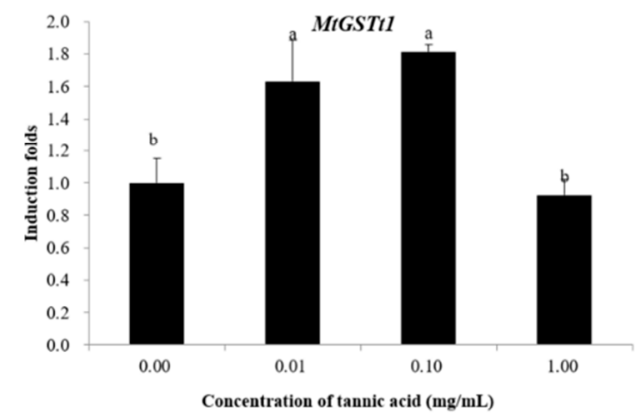

(d)

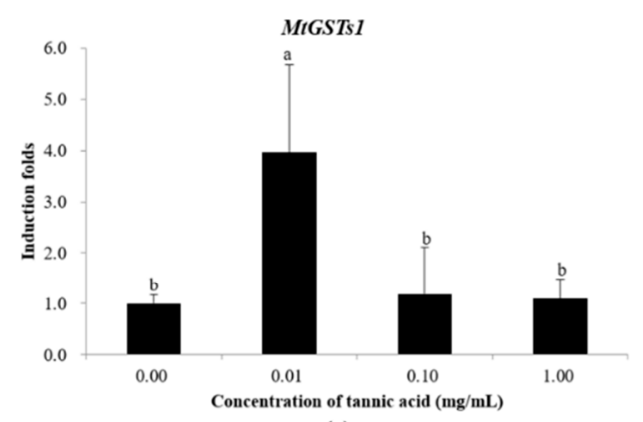

(c)

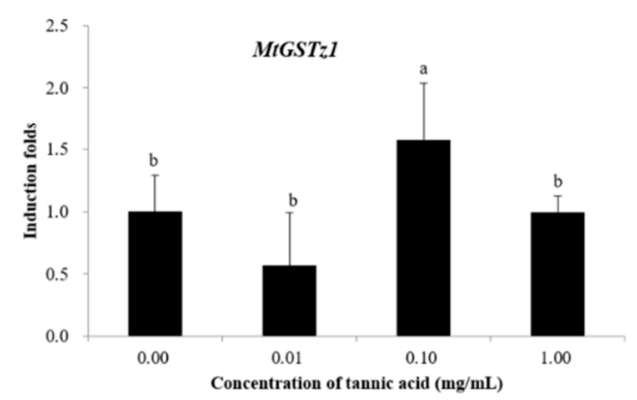

(e)

Figure 4. The effect of tannic acid on promoter activities. (a) MtGSTd2; (b) MtGSTo1; (c) MtGSTs1; (d) MtGSTt1; (e) MtGSTz1.

Promoter activities were measured as the luciferase activities in Sf9 cells. The normalized basal firefly luciferase activities (acetone control) were defined as 1 , and the normalized basal firefly luciferase activities (acetone control) were used as a positive control. The vertical bars indicate standard deviations of the mean $(n=4)$. The statistical significance of the induction folds was calculated using a one-way analysis of variance followed by Tukey's multiple comparisons, a value of $P<0.05$ was considered statistically significant.

\section{Discussion}

Based on their subcellular location, GSTs were classified into three categories: cytosolic GSTs, microsomal GSTs and mitochondrial GSTs [29]. Insect cytosolic GSTs were further divided into omega, sigma, epsilon, delta, thera and zeta classes based on amino acid sequences [30-32]. The five GSTs cloned from $M$. troglodyta in this study belonged to the omega, sigma, delta, thera and zeta classes. With the development of molecular biology techniques, more GST genes have been cloned and identified in insects [33-35], for example, the GST from the spruce budworm Choristoneura fumiferana (Clem.) [36,37] 
and the delta class GST from Drosophila melanogaster [29]. The acquisition of these GST genes laid the foundation for the study of GST characteristics and the regulation of GST expression in insects.

In this paper, five GST genes, MtGSTd2, MtGSTo1, MtGSTs1, MtGSTt1 and MtGSTz1, were cloned in M. troglodyta. Although we reported that MtGSTd1 mRNA expression was induced by tannic acid [19], the induction of GST activity by tannic acid was the overall result for all GST gene expression in vivo. Therefore, our results showed that the mRNA expression of four GST genes, MtGSTd2, MtGSTo1, MtGSTs1 and MtGSTz1, was also induced by tannic acid. These results were consistent with those of other studies. For example, Feng et al. reported that balsam fir foliage induced the expression of CfGST mRNA in Choristoneura fumiferana [36], and gstD1 and gstD21 mRNA expression in D. melanogaster was induced by phenobarbita [38]. These results showed that the increase in GST activities was mainly due to changes at the transcriptional level.

Plant secondary metabolites are inducers of insect GSTs in vivo. An increase in aphid GST activity was found in response to phenolic acids [39]; the GST activities of midguts were significantly induced by geranium petals and quisqualic acid in Popillia japonica Newman [40]; the GST activities were induced by quercetin in the silkworm [41]. This induction process appears to be an adaptation mechanism of organisms to counter chemical stress. Tannic acid is characterized as a plant polyphenol and is commonly distributed in many plants. Our previous results suggested that GST activities in insects were induced by feeding upon or exposure to tannic acid [19], which was in agreement with the response of insect herbivores to plant allelochemicals including the induction of GST activity by tannic acid in H. armigera [23]. We found that the increase in GST activity was mainly due to the increased expression of GST mRNA. However, little information is available on the molecular mechanisms by which GST mRNA expression is induced by tannic acid in insects. The isolation and characterization of promoters is helpful for the study of gene expression and regulation and provides basic materials for functional studies. However, there are no reports on GST promoters in M. troglodyta at home and abroad. Therefore, we studied the GST promoters of M. troglodyta for the first time. In this paper, the five promoter sequences of the five MtGST genes were cloned, which was very important in clarifying the molecular mechanisms of GST expression and regulation in M. troglodyta.

Plant secondary metabolites have an inhibitory effect on GST activities in vitro. For examples, Tang et al. showed that tannic acid inhibited GST activities in M. troglodyta and Clostera anachoreta (Fabricius) [21]; Tang et al. reported that GST activities from Odontotermes formosanus (Shiraki) and Reticulitermes chinensis Snyder were inhibited by tannic acid [42]. In addition, the rat liver GST was inhibited by plant polyphenols [20]; GSTs were inhibited by allelochemicals in cotton bollworm, H. armigera [43]; GSTs were inhibited by allelochemicals in the fall armyworm, Spodoptera frugiperda [44]. However, in this experiment, the restructured plasmids were transferred into Sf9 cells by lipofectin-mediated transfection, and luciferase assays were performed in the presence of tannic acid. The results showed that the GST promoter activities were induced by tannic acid. The difference may be due to one in vitro reaction and one in vivo reaction. In in vitro inhibition of GST activities, plant secondary metabolites react directly with GST protein; in in vivo induction of GST promoter activities, tannic acid need to enter Sf9 cells to react with GST promoters.

Currently, promoters are a research hotspot in genetic studies as some of the most important cis-acting elements in gene expression and regulation. Many inducible promoters have been identified in attempting to understand the molecular mechanisms of expression and regulation. A stress-induced gene (ZmRXO1) promoter was cloned and analysed in maize [45]. Fujita et al. found that the $B m N P V$ ie-1 promoter was involved in gene expression in various organisms, including insects, mammals, plants and bacteria [46]. Moreover, many functional elements and coregulatory binding sites for the nucleus were discovered. For example, $\mathrm{kB}$ and GATA factors alone were not sufficient to activate moricin expression in Manduca sexta, and the kB-GATA element from the Ms moricin promoter could significantly increase the activities of Drosophila melanogaster AMP gene promoters. Furthermore, a moricin promoter activating element (MPAE), which may contain coregulatory binding sites for nuclear factors specifically expressed in lepidopteran species, could increase the activity of the 
drosomycin promoter [47]. The transcription factor Sp1 was shown to promote human LRRK2 gene promoter activity and gene expression, whereas its inhibitor MTM reduced promoter activity and gene expression [48]. However, little information is available on the GST promoter in insects. Chen et al. showed that the Slgste1 promoter played an important role in regulating gene expression in response to phytochemicals and insecticides in Spodoptera litura [17]; Hu et al. reported that in the promoter region of the SeGST gene from Spodoptera exigua, cis-acting elements responded to chlorpyrifos and cypermethrin [18]. However, there have been no previous reports on the GST promoter in Notodontidae insects. In this paper, five GST gene promoters were isolated, and the activities of these five promoters under tannic acid stress were studied in M. troglodyta. The functional analysis results indicated that the promoter activities of MtGSTd2, MtGSTo1, MtGSTz1 and MtGSTs1 were induced by tannic acid, which was consistent with the transcriptional expression of these genes. These results showed that tannic acid could influence the activities of the GST promoters, and the GST promoters further regulated the transcriptional expression of GST genes in M. troglodyta. In addition, the promoter activity of MtGSTt1 was induced by tannic acid, while the transcriptional expression of MtGSTt1 was inhibited, which showed that in addition to the promoter, there may be negative regulatory elements involved in the expression of MtGSTt1 in M. troglodyta. Therefore, in future studies, we will focus on the core elements and response elements to verify the function of MtGST promoters and determine the regulatory mechanism controlling expression.

\section{Conclusions}

In summary, five GST genes were identified from M. troglodyta. These five GST genes were classified into five different cytosolic GST classes: delta, omega, sigma, theta, and zeta. The present study provided an overview of the five GST expression profiles under tannic acid stress in insect midguts and fat bodies. Moreover, the five MtGST promoters were isolated and analysed, which was firstly obtained in Notodontidae insects. Furthermore, the functional analysis results indicated that all four MtGST promoter activities, i.e., those of MtGSTd2, MtGSTo1, MtGSTz1 and MtGSTs1, were induced by tannic acid, which was consistent with the transcriptional expression of these genes, showing that the effects of tannic acid on the mRNA expression of GST genes as a result of promoter activity varied. However, the promoter activity of MtGSTt1 was induced by tannic acid, while the transcriptional expression of MtGSTt1 was inhibited, which showed that in addition to the promoter, there may be negative regulatory elements involved in the expression of MtGSTt1 in M. troglodyta. These results provide an important theoretical basis for elucidating the mechanism regulating tannic acid effects on the expression of GSTs. This will aid in deepening the understanding of the interactions between GST and tannic acid in M. troglodyta and is highly significant for the comprehensive management of this pest.

Supplementary Materials: The following materials are available online at http://www.mdpi.com/2075-4450/11/6/ 339/s1: Figure S1: MtGSTd2 promoter and part of the $5^{\prime}$ end of the coding sequence, Figure S2: MtGSTo1 promoter and part of the $5^{\prime}$ end of the coding sequence, Figure S3: MtGSTs1 promoter and part of the $5^{\prime}$ end of the coding sequence, Figure S4: MtGSTt1 promoter and part of the $5^{\prime}$ end of the coding sequence, Figure S5: MtGSTz1 promoter and part of the $5^{\prime}$ end of the coding sequence, Table S1: The optimal alignment of cloned sequences for Blastp, Table S2: The accession numbers for the other sequences included in the phylogenetic analyses.

Author Contributions: Conceptualization, F.T.; Formal analysis, H.T.; Funding acquisition, F.T.; Investigation, H.T.; Methodology, F.T., Q.S., X.G. and P.L.; Project administration, F.T.; Supervision, F.T.; Writing-original draft, F.T. and H.T.; Writing-review and editing, F.T. All authors have read and agreed to the published version of the manuscript.

Funding: This research was supported by the National Natural Science Foundation of China (Contract No. 31370652, 30600476 and 30972376), the National Natural Science Foundation of Jiangsu (Contract No.BK20151517), the China Postdoctoral Science Special Foundation (2014T70531), the General Financial Grant from the China Postdoctoral Science Foundation (2013M530262) and a project funded by the Priority Academic Program Development of Jiangsu Higher Education Institutions.

Acknowledgments: We thank the three anonymous reviewers for their helpful comments and suggestions for improving the manuscript. 
Conflicts of Interest: The authors declare no conflicts of interest.

\section{References}

1. Todaro, L.; Russo, D.; Cetera, P.; Milella, L. Effects of thermo-vacuum treatment on secondary metabolite content and antioxidant activity of poplar (Populus nigra L.) wood extracts. Ind. Crop. Prod. 2017, 109, 384-390. [CrossRef]

2. Berenbaum, M.R. The chemistry of defense: Theory and practice. Proc. Natl. Acad. Sci. USA 1995, 92, 2-8. [CrossRef] [PubMed]

3. Boyland, E.; Chasseaud, L.F. The role of glutathione and glutathione S-transferases in mercapturic acid biosynthesis. Adv. Enzymol. Relat. Areas. Mol. Biol. 1969, 32, 173-219. [PubMed]

4. Habig, W.H.; Pabst, M.J.; Jakoby, W.B. Glutathione S-transferases. J. Biol. Chem. 1974, 249, 7130-7139. [PubMed]

5. Morel, F.; Rauch, C.; Petit, E.; Piton, A.; Theret, N.; Coles, B.; Guillouzo, A. Gene and protein characterization of the human glutathione S-transferase Kappa and evidence for a peroxisomal localization. J. Biol. Chem. 2004, 279, 16246-16253. [CrossRef] [PubMed]

6. Hayes, J.D.; Flanagan, J.U.; Jowsey, I.R. Glutathione Transferases. Annu. Rev. Pharmacol. Toxicol. 2005, 45, 51-88. [CrossRef]

7. Feng, Q.L.; Davey, K.G.; Pang, A.S.D.; Ladd, T.M.; Retnakaran, A.; Tomkins, B.L.; Zheng, S.C.; Palli, S.R. Developmental expression and stress induction of glutathione S-transferase in the spruce budworm, Choristoneura fumiferana. J. Insect Physiol. 2001, 47, 1-10. [CrossRef]

8. Enayati, A.A.; Ranson, H.; Hemingway, J. Insect glutathione transferases and insecticide resistance. Insect Mol. Biol. 2005, 14, 3-8. [CrossRef]

9. McLellan, L.I.; Wolf, C.R. Glutathione and glutathione-dependent enzymes in cancer drug resistance. Drug Resist. Update 1999, 2, 153-164. [CrossRef]

10. Lee, K. Glutathione S-transferase activities in phytophagous insects: Induction and inhibition by plant phototoxins and phenols. Insect Biochem. 1991, 21, 353-361. [CrossRef]

11. Gao, X.W.; Dong, X.L.; Zheng, B.Z.; Chen, Q. Glutathiones S-transferase (GSTS) of cotton bollworm: Induction of pesticides and plant secondary substances and metabolism of GSTS to pesticides. Acta Entomol. Sin. 1997, 40, 122-127.

12. Robert, J.A.; Pitt, C.; Bonnett, T.R.; Yuen, M.M.S.; Keeling, C.I.; Bohlmann, J. Disentangling detoxifification: Gene expression analysis of feeding Mountain pine beetle illuminates molecular-level host chemical defense detoxifification mechanisms. PLoS ONE 2013, 8. [CrossRef] [PubMed]

13. Pitt, C.; Robert, J.A.; Bonnett, T.R.; Keeling, C.I.; Bohlmann, J.; Huber, D.P.W. Proteomics indicators of the rapidly shifting physiology from whole mountain pine beetle, Dendroctonus ponderosae (Coleoptera: Curculionidae), adults during early host colonization. PLoS ONE 2014, 9. [CrossRef]

14. Dai, L.L.; Ma, J.N.; Ma, M.Y.; Zhang, H.Q.; Shi, Q.; Zhang, R.R.; Chen, H. Characterisation of GST genes from the Chinese white pine beetle Dendroctonus armandi (Curculionidae: Scolytinae) and their response to host chemical defence. Pest Manag. Sci. 2016, 72, 816-827. [CrossRef]

15. Huang, Y.F.; Xu, Z.B.; Lin, X.Y.; Feng, Q.L.; Zheng, S.C. Structure and expression of glutathione S-transferase genes from the midgut of the common cutworm, Spodoptera litura (Noctuidae) and their response to xenobiotic compounds and bacteria. J. Insect Physiol. 2011, 57, 1033-1044. [CrossRef] [PubMed]

16. Li, Q.L.; Xie, J.H.; Ma, X.Q.; Li, D. Molecular cloning of Phosphoethanolamine N-methyltransferase (PEAMT) gene and its promoter from the halophyte Suaeda liaotungensis and their response to salt stress. Acta Physiol. Plant 2016, 38. [CrossRef]

17. Chen, S.; Lu, M.; Zhang, N.; Zou, X.; Mo, M.; Zheng, S. Nuclear factor erythroid-derived 2-related factor 2 activates glutathione S-transferase expression in the midgut of Spodoptera litura (Lepidoptera: Noctuidae) in response to phytochemicals and insecticides. Insect Mol. Biol. 2018, 27, 522-532. [CrossRef] [PubMed]

18. Hu, B.; Huang, H.; Wei, Q.; Ren, M.M.; Mburu, D.K.; Tian, X.R.; Su, J.Y. Transcription factors CncC/Maf and AhR/ARNT coordinately regulate the expression of multiple GSTs conferring resistance to chlorpyrifos and cypermethrin in Spodoptera exigua. Pest Manag. Sci. 2019, 75, 2009-2019. [CrossRef]

19. Cheng, H.P.; Tang, F.; Li, W.; Xu, M. Tannic acid induction of a glutathione S-transferase in Micromelalopha troglodyta (Lepidoptera: Notodontidae) larvae. J. Entomol. Sci. 2015, 50, 350-362. [CrossRef] 
20. Zhang, K.; Das, N.P. Inhibitory effects of plant polyphenols on rat liver glutathione S-transferases. Biochem. Pharmacol. 1994, 47, 2063-2068. [CrossRef]

21. Tang, F.; Zhang, X.B.; Liu, Y.S.; Gao, X.W.; Liu, N.N. In vitro inhibition of glutathione S-transferases by several insecticides and allelochemicals in two moth species. Int. J. Pest Manag. 2014, 60, 33-38. [CrossRef]

22. Yamamoto, K.; Zhang, P.B.; Miake, F.; Kashige, N.; Aso, Y.; Banno, Y.; Fujii, H. Cloning, expression and characterization of theta-class glutathione S-transferase from the silkworm, Bombyx mori. Comp. Biochem. Physiol. 2005, 141, 340-346. [CrossRef]

23. Chen, F.J.; Gao, X.W.; Lei, M.Q.; Zheng, B.Z. Effects of tannic acid on glutathione S-transferases in Helicoverpa armigera (Hübner). Acta Entomol. Sin. 2003, 46, 684-690.

24. Tang, F.; Zhang, X.B.; Liu, Y.S.; Gao, X.W. Tissue distribution and properties of glutathione S-transferases in Micromelalopha troglodyta (Lepidoptera: Notodontidae). J. Entomol. Sci. 2008, 43, 268-278. [CrossRef]

25. Zhang, X.B.; Tang, F.; Liu, Y.S.; Gao, X.W. Induction of glutathione S-transferases by tannic acid in Micromelalopha troglodyta. Chin. Bull. Entomol. 2009, 46, 579-584.

26. Tamura, K.; Stecher, G.; Peterson, D.; Filipski, A.; Kumar, S. MEGA6: Molecular evolutionary genetics analysis version 6.0. Mol. Biol. Evol. 2013, 30, 2725-2729. [CrossRef] [PubMed]

27. Giulietti, A.; Oververgh, L.; Valckx, D.; Decallonne, B.; Bouillon, R.; Mathieu, C. An overview of real-time quantitative PCR: Applications to quantify cytokine gene expression. Methods 2001, 25, 386-401. [CrossRef] [PubMed]

28. Peng, T.; Pan, Y.; Gao, X.; Xi, J.; Zhang, L.; Yang, C.; Bi, R.; Yang, S.; Xin, X.; Shang, Q. Cytochrome P450 CYP6DA2 regulated by cap ' $\mathrm{n}$ 'collar isoform $\mathrm{C}(\mathrm{CncC})$ is associated with gossypol tolerance in Aphis gossypii Glover. Insect Mol. Biol. 2016, 25, 450-459. [CrossRef]

29. Agianian, B.; Tucker, P.A.; Schouten, A.; Leonard, K.; Bullard, B.; Gros, P. Structure of a drosophila sigma class glutathione $\mathrm{S}$-transferase reveales a novel active site topography suited for lipid peroxidation products. J. Mol. Biol. 2003, 326, 151-165. [CrossRef]

30. Pemble, S.E.; Taylor, J.B. An evolutionary perspective on glutathione transferases inferred from class-theta glutathione transferase cDNA sequences. Biochem. J. 1992, 287, 957-963. [CrossRef]

31. Ranson, H.; Cornel, A.J.; Fournier, D.; Vaughan, A.; Collins, F.H.; Hemingway, J. Cloning and localization of a glutathione S-transferase classIgene from Anopheles gambiae. J. Biol. Chem. 1997, 272, 5464-5468. [CrossRef] [PubMed]

32. Board, P.G.; Baker, R.T.; Chelvanayagam, G.; Jermiin, L.S. Zeta, a novel class of glutathione transferases in a range of species from plants to humans, Biochem. J. 1997, 328, 929-935.

33. Toung, Y.P.; Hsieh, T.S.; Tu, C.P. The glutathione S-transferase D genes: A divergently organized, intronless gene family in Drosophila melanogaster. J. Biol. Chem. 1993, 268, 9737-9746.

34. Yu, Q.Y.; Lu, C.; Li, B.; Fang, S.M.; Zuo, W.D.; Dai, F.Y.; Zhang, Z.; Xiang, Z.H. Identification, genomic organization and expression pattern of glutathione S-transferase in the silkworm, Bombyx mori. Insect Biochem. Mol. 2008, 38, 1158-1164. [CrossRef] [PubMed]

35. Ding, Y.C.; Ortelli, F.; Rossiter, L.C.; Hemingway, J.; Ranson, H. The Anopheles gambiae glutathione transferase supergene family: Annotation, phylogeny and expression profiles. BMC Genom. 2003, 35. [CrossRef] [PubMed]

36. Feng, Q.L.; Davey, K.G.; Pang, A.S.; Primavera, M.; Ladd, T.R.; Zheng, S.C.; Sohi, S.S.; Retnakaran, A.; Palli, S.R. Glutathione S-transferase from the spruce budworm, Choristoneura fumiferana: Identification, characterization, localization, cDNA cloning, and expression. Insect Biochem. Mol. 1999, 29, 779-793. [CrossRef]

37. Zheng, S.C.; Deng, H.M.; Ladd, T.; Tomkins, B.L.; Krell, P.J.; Feng, Q.L. Cloning and characterization of two glutathione S-transferase cDNAs in the Spruce Budworm, Choristoneura fumifera. Arch. Insect Biochem. Physiol. 2007, 66, 146-157. [CrossRef]

38. Tang, A.H.; Tu, C.P. Pentobarbital-induced changes in Drosophila glutathione S-transferase D21 mRNA stability. J. Biol. Chem. 1995, 270, 13819-13825. [CrossRef]

39. Chrzanowski, G.; Leszczynski, B.; Czerniewicz, P.; Sytykiewicz,H.; Matok, H.; Krzyzanowski, R.; Sempruch, C. Effect of phenolic acids from black currant, sour cherry and walnut on grain aphid (Sitobion avenae F.) development. Crop Prot. 2012, 35, 71-77. [CrossRef]

40. Adesanya, A.W.; Held, D.W.; Liu, N.N. Geranium intoxication induces detoxification enzymes in the Japanese beetle, Popillia japonica Newman. Pestic. Biochem. Phys. 2017, 143, 1-7. [CrossRef]

41. Zhang, Y.E.; Ma, H.J.; Feng, D.D.; Lai, X.F.; Chen, Z.M.; Xu, M.Y.; Yu, Q.Y.; Zhang, Z. Induction of detoxification enzymes by quercetin in the silkworm. J. Econ. Entomol. 2012, 105, 1034-1042. [CrossRef] [PubMed] 
42. Tang, F.; Zhu, T.; Gao, X.W.; Yan, A.J. Inhibition of glutathione S-transferases activity from Odontotermes formosanus Shiraki and Reticulitermes chinensis Snyder by seven inhibitors. Acta Entomol. Sin. 2007, 50, $1225-1231$.

43. Chen, F.J.; Zhang, C.Z.; Gao, X.W. In vitro inhibition of glutathione S-transferases by several insecticides and allelochemicals in cotton bollworm, Helicoverpa armigera Hübner. J. Entomol. Sci. 2007, 42, $296-305$. [CrossRef]

44. Yu, S.J.; Abo-Elghar, G.E. Allelochemicals as inhibitors of glutathione S-transferases in the fall armyworm. Pestic. Biochem. Phys. 2000, 68, 173-183. [CrossRef]

45. Tao, Y.; Wang, F.T.; Jia, D.M.; Li, J.T.; Zhang, Y.M.; Jia, C.G.; Wang, D.P.; Pan, H.Y. Cloning and functional analysis of the promoter of a stress-inducible gene (ZmRXO1) in Maize. Plant Mol. Biol. Rep. 2015, 33, 200-208. [CrossRef]

46. Fujita, R.; Ono, C.; Ono, I.; Asano, S.; Bando, H. Analysis of the Bombyx mori nucleopolyhedrovirus ie-1 promoter in insect, mammalian, plant, and bacterial cells. Biochem. Biophys. Res. Commun. 2015, 464, 1297-1301. [CrossRef]

47. Rao, X.J.; Xu, X.X.; Yu, X.Q. Mandnca sexta moricin promoter elements can increase promoter activities of Drosophila melanogaster antimicrobial peptide genes. Insect Biochem. Mol. 2011, 41, 982-992. [CrossRef]

48. Wang, J.L.; Song, W.H. Regulation of LRRK2 promoter activity and gene expression by Sp1. Mol. Brain 2016, 9. [CrossRef]

(C) 2020 by the authors. Licensee MDPI, Basel, Switzerland. This article is an open access article distributed under the terms and conditions of the Creative Commons Attribution (CC BY) license (http://creativecommons.org/licenses/by/4.0/). 